\title{
Design of sEMG Acquisition Circuit and Its Adaptive EEMD Denosing Research
}

\author{
Wei $\mathrm{Li}^{1}$, Wei $\mathrm{Hu}^{2}$, Kun $\mathrm{Hu}^{3}$, Qiang Qin ${ }^{1 *}$ \\ ${ }^{1}$ School of Advanced Manufacturing Engineering of Hefei University, Hefei, 230601, China \\ ${ }^{2}$ The $43^{\text {rd }}$ Research Institute of China Electronics Technology Group Corporation, Hefei, 230071, China \\ ${ }^{3}$ School of Automation, Northwestern Polytechnical University, Xian, 710072, China
}

Received: June 8, 2021. Revised: December 16, 2021. Accepted: January 12, 2022. Published: January 14, 2022.

\begin{abstract}
The Surface electromyography (sEMG) signal is a kind of electrical signal which generated by human muscles during contraction. It is prone to being affected by noise because of its small amplitude, so it is necessary to remove the noise in its original signal with an appropriate algorithm. Based on the traditional signal denoising indicators, a new complex indicator $r$ has been proposed in this paper which combines three different indicator parameters, that is, Signal to Noise Ratio (SNR), correlation coefficient $(R)$, and standard error (SE). At the same time, an adaptive ensemble empirical mode decomposition (EEMD) method named AIO-EEMD which based on the proposed indicator is represented later. To verify the effective of the proposed algorithm, an electromyography signal acquisition circuit is designed firstly for collecting the original sEMG signal. Then, the denosing performance from the designed method is been compared with empirical mode decomposition (EMD) method and wavelet transform noise reduction method, respectively. The experiment results shown that the designed algorithm can not only automatically get the numbers of the reconstructed signal numbers, but also obtain the best reduction performance.
\end{abstract}

Keywords-Surface electromyography, acquisition circuit, noise reduction method, AIO-EEMD, Optimum number of cycles

\section{INTRODUCTION}

$\mathrm{T}$ HE sEMG signal can be used to record and evaluate the electrical activity of human muscle fibers, and it is usually acquired by placing surface electrodes in the skin or inserting needle electrodes into the cells of specific muscles [1]. It has been widely used in clinical muscle disease diagnosis, sports medicine, rehabilitation engineering and many other fields [2], [3].

A multi-channel acquisition circuit which consisting of several common operational amplifier chips was designed for acquiring sEMG in this paper. The noise coupled into sEMG is relatively loud because of the low-cost operational amplifier (op-amp) selected in the acquisition circuit. These mixed noise that contains useful sEMG signal will converted into digital quantities by $\mathrm{A} / \mathrm{D}$ converter together with the useful signal, which will greatly affect the peak position and width of the original sEMG signal. Therefore, it is necessary to denoise the sEMG signal with certain reduction methods.

The most commonly method used for signal denoising is smoothing, i.e., the signal is averaged several times after adding Gaussian white noise to the original signal. The moving average method achieves the noise reduction target by averaging or fitting the points within a data window of width $2 r+1$. Due to the resolution problem, the signal distortion phenomenon will inevitably occur when the window has multiple data, on the contrary, the noise reduction will not reach its expected effect. To overcome the shortcomings of the moving average method, the convolutional noise reduction method was first proposed by Savitzky-Golay, which is an extension of moving average method [4]. In addition, the EMD algorithm is also one of the common methods, while it is easy to appear the modal aliasing problem [5]. To counteract the modal aliasing problem in signal decomposition process, a new method which combines EMD and wavelet packets was proposed by Liu [6]. The experiment results shown that the method is effective. The concept of noise index was put forward by Zhang and it is a fixed value which define as the ratio of the variance of $\mathrm{IMF}_{2} \sim \mathrm{IMF}_{\mathrm{n}}$ to IMF $\mathrm{IMfter}_{1}$ the signal is decomposed by EEMD [7]. The index can be used to estimate the variance of noise containing IMF components except $\mathrm{IMF}_{1}$, which can improve both the efficiency of the algorithm and the accuracy of the threshold estimation. Although the EEMD method can solve modal aliasing problem, the shortcoming of this method is that the parameters need to be set manually [8].

To solve the above problem, an adaptive EEMD noise reduction method based on Normalized Index Optimization (NIO) has been proposed in the paper. It introduced a new index $r$ which consists of signal to noise ratio (SNR), degree of 
approximation (SE) and relevance between each different signals (R). To verify the reliability of the proposed algorithm, a circuit used for collecting the EMG signal is designed firstly. Then, the proposed algorithm is used for denoising the acquired signals.

The paper is organized as follows. Section II describes the circuit that used for acquiring the sEMG signal. The proposed new algorithm is used to perform noise reduction experiment in Section III. Finally, the results of noise reduction experiment are analyzed and studied. The research content of this paper is shown in Fig. 1.

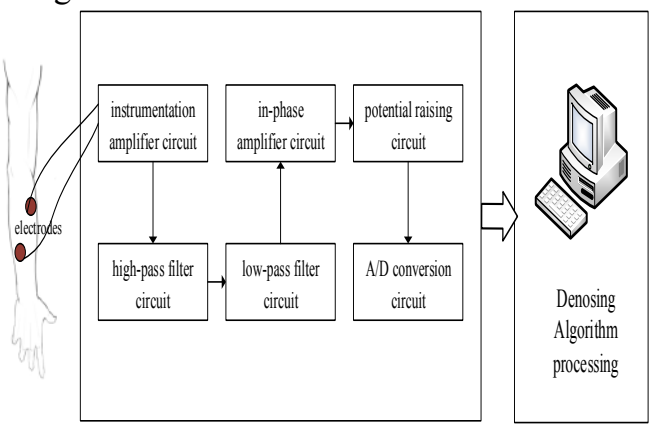

Figure 1. System Framework Diagram

\section{DESIGNING OF SEMG ACQUISITION CIRCUIT}

\section{A. Characteristics of sEMG}

The sEMG signal is a weak signal with small amplitude and typically in the order of tens of millivolts [9]. A variety of noises will coupled in the acquisition process of sEMG signal, such as baseline noise, motion artifact noise, ambient noise, high frequency noise generated by a circuit composed of multiple op-amps, etc [10], [11]. For the low frequency noise generated from the moving wires or fluctuating electrodes, it can be filtered out by using a band-pass filter or a high-pass filter. As for the high frequency noise of flicker noise and motion artifact noise which generated by the circuit composed of multiply op-amps, it can be reduced by selecting a suitable algorithm such as EEMD or wavelet to get useful signals.

\section{B. Overall structure of the sEMG acquisition circuit}

In order to acquire sEMG signal more accurately and suppress the electromagnetic noises, the values of both the common mode rejection ratio (CMRR) and SNR are required to be as large as possible. Therefore, how to increase the values of both the CMRR and SNR are the key problems in the design of sEMG acquisition circuit [11]. To fulfill the above requirements, a circuit designed for the system in this paper is described as follows.

The low-cost multi-channel sEMG signal acquisition circuit designed in our system mainly consists of three parts, that is, instrumentation amplifier circuit, frequency band filter circuit, and in-phase amplifier circuit. Among them, the instrumentation amplifier circuit can realize differential pre-amplification function by using three operational amplifiers [13], [14]. The instrumentation amplifier circuit contains two stages, i.e., the first stage with the characteristics of two-terminal differential input and two-terminal differential output, and the second stage with the characteristics of differential output and single-terminal input. To meet the frequency requirements, a band-pass filter is required to filter the signal obtained from the instrumentation amplifier circuit. It is usually appropriate to set the bandpass frequency of the circuit from $10 \mathrm{~Hz}$ to $500 \mathrm{~Hz}$ [15]. In this system, a second-order high-pass filter with low cutoff frequency of $16 \mathrm{~Hz}$ and a second-order low-pass filter with high-cutoff frequency of $482 \mathrm{~Hz}$ were cascaded to form a band-pass filter to fulfill the above requirements. When the low-pass filter and the high-pass filter are connected in series, a band-pass circuit is formed which can only pass the signal in the band between these two different thresholds. After the signal with a specific frequency band is obtained, the signal will be amplified by amplifier circuit. When the signal is amplified and filtered as described above, the sEMG signal needs to be further amplified in order to make the output voltage of sEMG match the voltage range of the $\mathrm{A} / \mathrm{D}$ converter on microcontroller. An in-phase op-amp circuit was adopted to achieve this function in the whole circuit, and the op-amp type is TL084. Finally, a satisfactory signal will be obtained after it passes through the above three stages and the whole circuit diagram system is shown in Fig. 2.

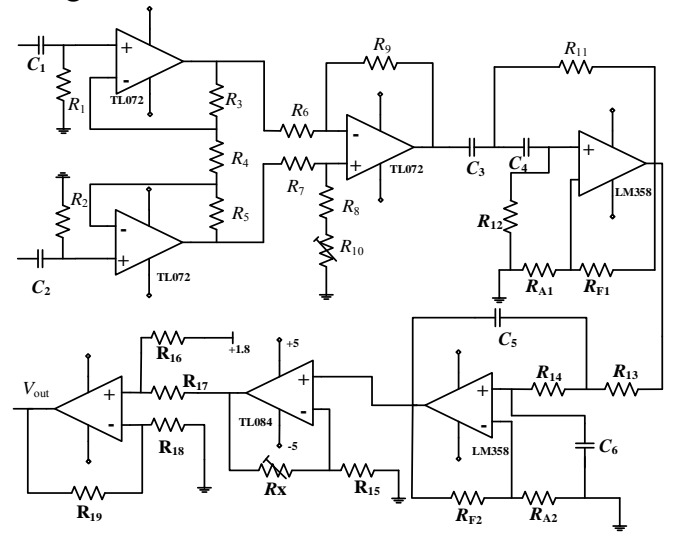

Figure 2. sEMG acquisition circuit schematic

\section{Analyzing of sEMG acquisition circuit}

To eliminate the noise signal and improve the CMRR, the chips widely used in commercial signal acquisition products on the market are generally integrated ADC chips. In [16], the chip used in the designed circuit was ADS1299. It has the disadvantage of costly and too powerful. To overcome the above problem, three low-cost op-amps were adopted in the instrumentation amplifier in sEMG acquisition circuit in this paper. The instrumentation amplifier performs the differential pre-amplification function based on the differential amplifier principle, which is useful for handling the noise of such a weak signal like sEMG. The above three op-amps from a two-stage instrumentation amplifier circuit. What's more, a Field Effect Transistor (FET) input op-amp was added in the circuit 
because of the large impedance in the electrodes, and the model of the op-amp was TL072. To facilitate adjustment and obtain a better amplification effect, the values of the input op-amp impedance in each stage were equal. In the post amplification stage, an adjustable resistor R10 was connected in series with R8. When R9/R6 equals to R8/R7, the CMRR reached its maximum value. In order to simplify the designed circuit and realize the band-pass filter circuit function, a high-pass filter circuit and a low-pass filter circuit were combined together in our system. Meanwhile, keeping $\mathrm{R} 11=\mathrm{R} 12=\mathrm{R} \& \mathrm{C} 3=\mathrm{C} 4=\mathrm{C}$ in the high-pass filter circuit and $\mathrm{R} 13=\mathrm{R} 14=\mathrm{R}^{\prime} \& \mathrm{C} 5=\mathrm{C} 6=\mathrm{C}^{\prime}$ in the low-pass filter circuit. The low cutoff frequency in high-pass filter circuit and high cutoff frequency in low-pass filter circuit were calculated by equation (1) and equation (2), respectively.

$$
\begin{aligned}
& \left\{\begin{array}{l}
\omega_{\mathrm{n}}=\frac{1}{\sqrt{R_{11} R_{12} C_{3} C_{4}}} \\
f_{\mathrm{L}}=\frac{\omega_{\mathrm{n}}}{2 \pi}=\frac{1}{2 \pi R C}
\end{array}\right. \\
& \left\{\begin{array}{l}
\omega_{\mathrm{n}}=\frac{1}{\sqrt{R_{13} R_{14} C_{5} C_{6}}} \\
f_{\mathrm{H}}=\frac{\omega_{\mathrm{n}}}{2 \pi}=\frac{1}{2 \pi R^{\prime} C^{\prime}}
\end{array}\right.
\end{aligned}
$$

And the gain Av is represented by equation (3).

$$
A_{\mathrm{v}}=1+\frac{R_{\mathrm{Fi}}}{R_{\mathrm{Ai}}} \quad \mathrm{i} \in(1,2)
$$

It is finally determined that the low cutoff frequency of the high pass filter circuit is $16 \mathrm{~Hz}$ and the high cutoff frequency of the low pass filter circuit is $482 \mathrm{~Hz}$. With this design, the entire band-pass filter can obtain sEMG signal in the frequency range of $20 \mathrm{~Hz}$ to $500 \mathrm{~Hz}$. To ensure the signal can be transmitted to the microcontroller in a digital form and meet the demands of the $A / D$ conversion circuit in the microcontroller, an in-phase amplifying circuit and potential raising circuit were both added in the system. The specific design of the two circuits were not described in detail here, and the final acquired sEMG signal transmitted to the microcontroller were all positive digital signals.

\section{ADAPTIVE EEMD NOISE REDUCTION METHOD BASED ON NIO}

\section{A. Principle of EEMD}

As an improvement of EMD method, the EEMD method is more efficient and simple. The decomposition steps of EEMD method are as follows [17], [18].

First, the Gaussian white noise with same length but different amplitude is superimposed on the original signal, and then decomposed the superimposed mixed signal with EMD method[19], [20]. The cycle numbers of decomposition process is represented by $\mathrm{k}$. There will be an IMF component after each decomposition and its corresponding component can be represents as $\mathrm{IMF}_{\mathrm{ik}}$. The value of the superimposed Gaussian white noise is shown by Equation (4).

lne $+0.5 \mathrm{alnk}=0$

Where, e is the standard deviation which used for indicating the deviation result of the original sEMG from the EMD algorithm, and a represented the amplitude of the superimposed white noise.

Second, take the overall average of the IMF components obtained by EEMD decomposition and its calculation formula can be expressed as.

$I M F_{\mathrm{i}}=\frac{1}{\mathrm{n}} \sum_{\mathrm{k}=1}^{\mathrm{n}} I M F_{\mathrm{ik}}$

Where, $\mathrm{n}$ is the decomposition cycle numbers from EMD. After obtaining the IMF components with different frequencies, a low-pass filter needs to be constructed to filter out the high-frequency noise.

\section{B. Design of adaptive EEMD noise reduction method based on NIO}

In the denosing process with ordinary EEMD algorithm, the number of denosing cycles is usually determined by artificial experience [21]-[24]. Since the algorithm only focus on the improvement of SNR, it may not achieve the best denosing effect. And the biggest disadvantage of this algorithm is that it needs the help of manual setting for the parameters in the whole process. To deal with this problem, an adaptive algorithm, which can realize the target of determination the numbers of IMF components with the designed index was proposed in this paper. It can adaptively judge whether the noise reduction is optimal. During the noise reduction procedure, a normalized noise reduction index $r$ that used for evaluate the noise reduction performance is created by adopting the following three parameters, SNR, correlation coefficient (R), and standard error (SE). The detail formulas of the four indexes are as follows.

$$
\begin{aligned}
& S N R=10 \log \frac{\mathrm{S}}{\mathrm{N}}=10 \log \left[\frac{\sum_{\mathrm{j}=1}^{\mathrm{n}} x_{\mathrm{i}+1}^{2}(\mathrm{j})}{\sum_{\mathrm{j}=1}^{\mathrm{n}}\left[x_{\mathrm{i}}(\mathrm{j})-x_{\mathrm{i}+1}(\mathrm{j})\right]^{2}}\right] \\
& S E=\sqrt{\frac{1}{\mathrm{n}} \sum_{\mathrm{j}=1}^{\mathrm{n}}\left[x_{\mathrm{i}}(\mathrm{j})-x_{\mathrm{i}+1}(\mathrm{j})\right]^{2}} \\
& R=\frac{\left.\sqrt{\sum_{\mathrm{j}=1}^{\mathrm{n}}\left(x_{\mathrm{i}}(\mathrm{j})-\overline{x_{\mathrm{i}}}\right)\left(x_{\mathrm{i}+1}(\mathrm{j})-\overline{x_{\mathrm{i}+1}}\right)}\right)^{2} \sqrt{\sum_{\mathrm{j}=1}^{\mathrm{n}}\left(x_{\mathrm{i}+1}(\mathrm{j})-\overline{x_{\mathrm{i}+1}}\right)^{2}}}{r=\left|\frac{\mathrm{SNR}^{\prime}-\mathrm{SNR}}{\mathrm{SNR}}+\frac{1}{\mathrm{SE}}+\mathrm{R}\right|}
\end{aligned}
$$

Where, SNR represents the Signal to Noise Ratio of the original sEMG signal, SNR' represents the SNR of the signal after denoising. The designing of $r$ ensures that all the three 
items are all normalized. It can not only taking into account the importance of the three parameters, but also making their weights at a same level. After that, a new adaptive indicator optimizing EEMD (AIO-EEMD) algorithm has been designed with the indicator $r$ and its flowchart is shown in Fig. 3.

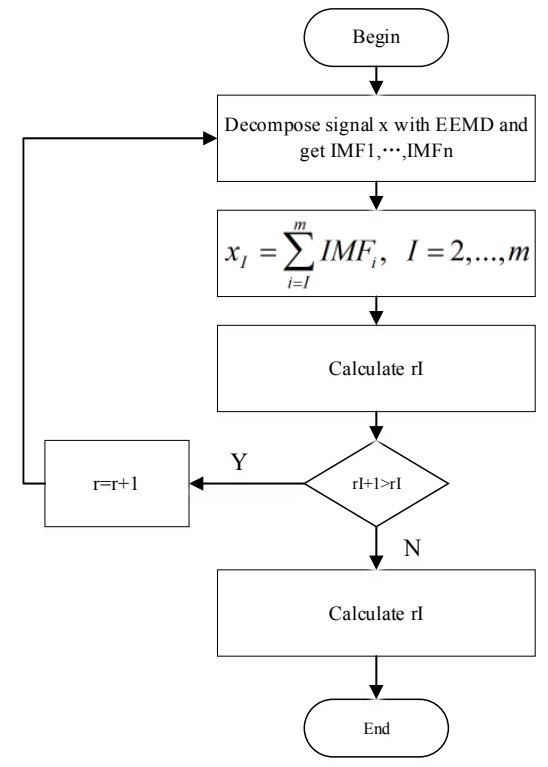

Figure 3. Noise Reduction Flowchart of AIO-EEMD

The detailed noise reduction steps of AIO-EEMD are as follows.

Step 1: Decompose the original sEMG signal to obtain the components from IMF1 to IMFm with the EEMD method.

Step 2: Remove the highest frequency component from all the IMF components and the remaining are combined together as XI. EEMD decomposition and reconstruction of signal XI are carried out once more according to the formal two steps to obtain signal $\mathrm{xI}+1$.

Step 3: Compare the values of $\mathrm{rI}$ and $\mathrm{rI}+1$, if $\mathrm{rI}+1$ is larger than $\mathrm{rI}$, it means that the signal reconstructed with $\mathrm{I}+1$ to $\mathrm{m}$ is better than the one with I to $\mathrm{m}$. So the signal can be further improved by removing the next IMF component. Then, let $\mathrm{I}=\mathrm{I}+1$, and the signal continues to be reconstructed until $\mathrm{rI}+1$ is smaller than rI, which means reconstructed sEMG signal with AIO-EEMD has reached the best noise reduction.

\section{EXPERIMENT}

\section{A. Experiment and result analysis of sEMG acquisition}

The designed sEMG acquisition circuit can not only acquire sEMG signal from human body under different channels, but also different movement signals in the same position under same channel. A voltage source and a $1 \mathrm{M} \Omega$ resistor in series were used in the formal acquisition circuit to simulate the electrodes impedance of the skin [24]. To verify the frequency response of the designed acquisition circuit, a sine wave of 1 $\mathrm{mV} / \mathrm{rms}$ with different frequencies $(0.1 \mathrm{~Hz}$ to $10 \mathrm{kHz})$ was used as the input signal for simulation. The measured values and phase margins corresponding to each frequency are shown in
Fig. 4. It can be seen that the measurement performance are accord with the design demands.

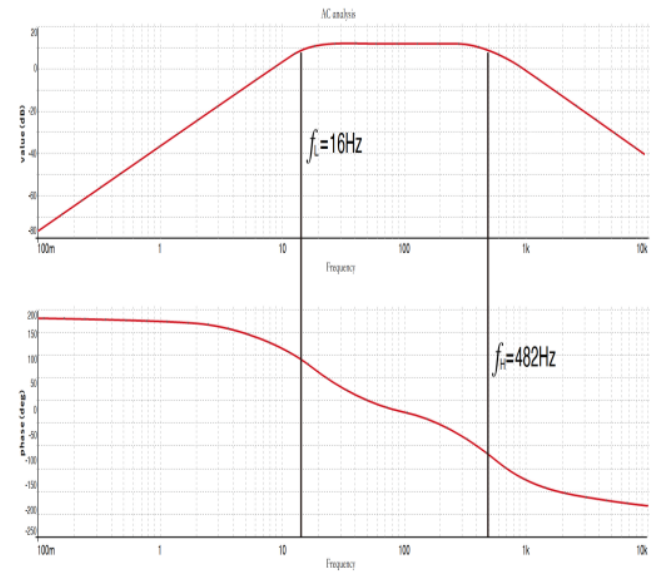

Figure 4. Frequency Characteristics of the Acquisition Circuit Filter

The sEMG signal was acquired by applying the two electrode pads to different positions on the lower arm. The fisting signal was acquired by channel 1 and the field experiment image is shown in Fig. 5.

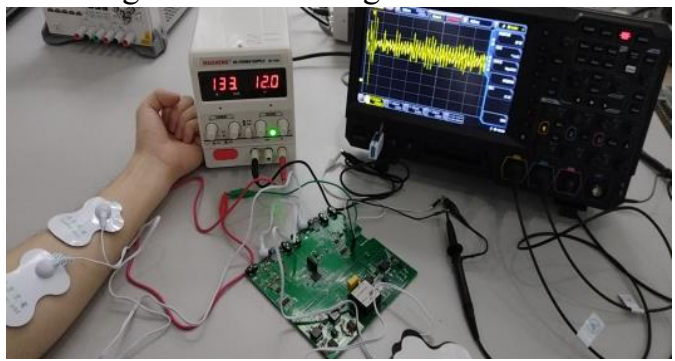

Figure 5. Image of Signal Acquisition Experiment

The output waveform from the sEMG acquisition circuit is obtained with an oscilloscope, and the detailed waveform from the experiment is shown in Fig. 6. The acquired sEMG signal will be further processed by A/D converter in STM32 microcontroller.

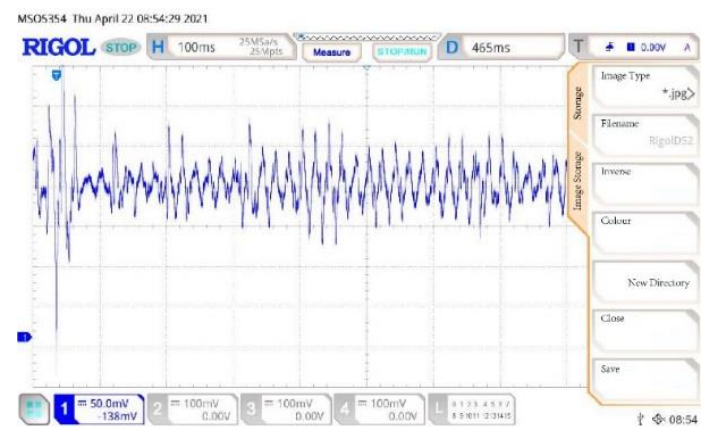

Figure 6. Detailed Waveform

It can be seen from Fig. 6 that the signal from sEMG acquisition circuit which composed of ordinary op amps will be coupled into much noise, and the final signal will coupled with a lot of burrs. Although the acquisition circuit designed in this paper can filter out the useless signals that less than $16 \mathrm{~Hz}$ or greater than $482 \mathrm{~Hz}$ with its bandpass filter circuit, the noise that are not in this frequency range cannot be filtered by using 
this analog circuit only. The noise without filtering will be mixed with the useful sEMG and converted to a digital quantify by $\mathrm{A} / \mathrm{D}$ converter, which will greatly affect the peak position and width of sEMG. Further, it will seriously affect the subsequent analysis and use of sEMG. Therefore, it is necessary to further denoise the collected raw sEMG.

\section{B. Experiment and result analysis of AIO- EEMD}

A corresponding MATLAB procedure was written based on the adaptive AIO-EEMD algorithm designed in Section III, and a group of sEMG signal was selected as the research object for noise reduction. The sampled signal contains 2100 sampling points. The amplitude standard deviation ratio coefficient of the white noise that added to signal is 0.25 , and the loop execution number $\mathrm{NE}$ of the program is 1 . After one EEMD decomposition performed on the signal, the system finally gets 11 IMF components, which are shown in Fig. 7.

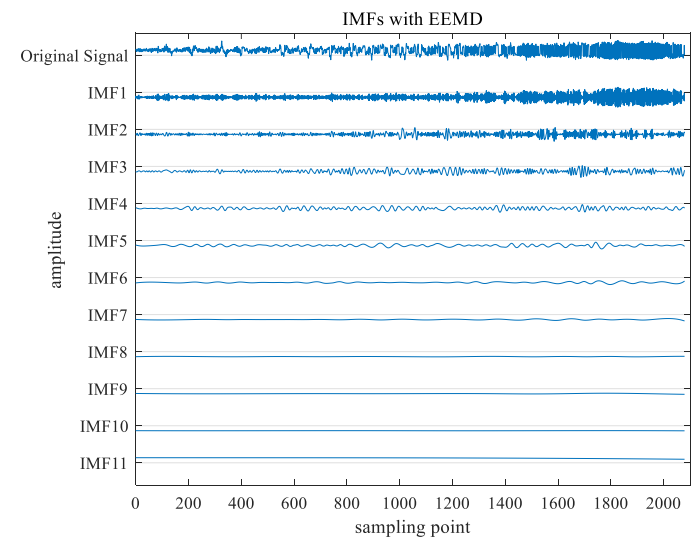

Figure 7. IMF Component Obtained by EEMD of Sample sEMG

As showed in Fig. 7, $\mathrm{IMF}_{1}$, as the highest frequency component of the noise, occupies a large amount of energy in the signal. For all those components, it is necessary to select appropriate components to reconstruct the signal. In order to determinate which IMFs to remove and which IMFs to retain for signal reconstruction, a comparative reconstruction experiment after removing different IMF components were conducted in this paper. The reconstruction results from removing $\mathrm{IMF}_{1}$ to $\mathrm{IMF}_{1}-\mathrm{IMF}_{6}$ are shown in Fig. 8., respectively.
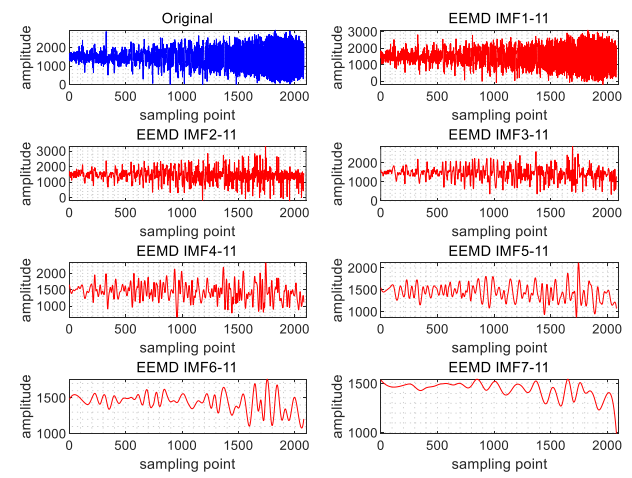

Figure 8. Signals Reconstructed using Different IMF Components
It can be seen that the effect of the reconstructed sEMG signal after only removing $\mathrm{IMF}_{1}$ is significantly better than other combinations. It can be seen that too much removal of IMF components will reduce the useful signal and lead to a reduction in amplitude of the reconstructed signal, or even signal distortion. It is relatively robust to adopt the strategy of removing only $\mathrm{IMF}_{1}$ in each EEMD denosing process in this paper. It will be automatically remove the noise in the next cycle if there is still noise retained in the current reconstructed signal through the proposed method, and the number of EEMD cycles will be finally decided by normalizing the optimization-seeking index. After determining the reconstructed components as $\mathrm{IMF}_{2}-\mathrm{IMF}_{11}$, the normalized index optimization adaptive EEMD denosing procedure is executed to conduct noise reduction experiments on fisting signal until $\mathrm{r}$ drops. The experiment results was shown in Fig. 9.

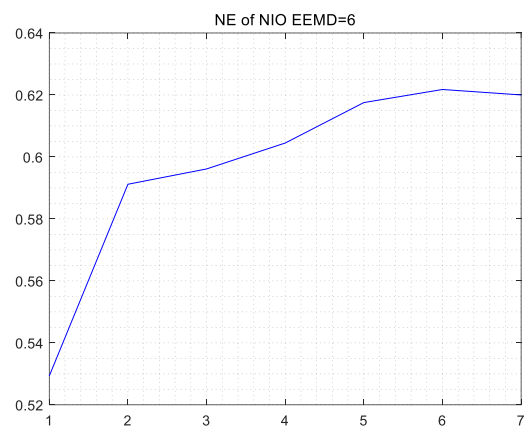

Figure 9. Normalized Optimization Index r Corresponding to Cycle Number

It can been seen from the curve that the maximum value of $r$ appeared when it equals to 6 , which means that the denosing performance corresponding to this moment was the best. The original signal curve and the reconstructed signal curve were shown in Fig. 10.

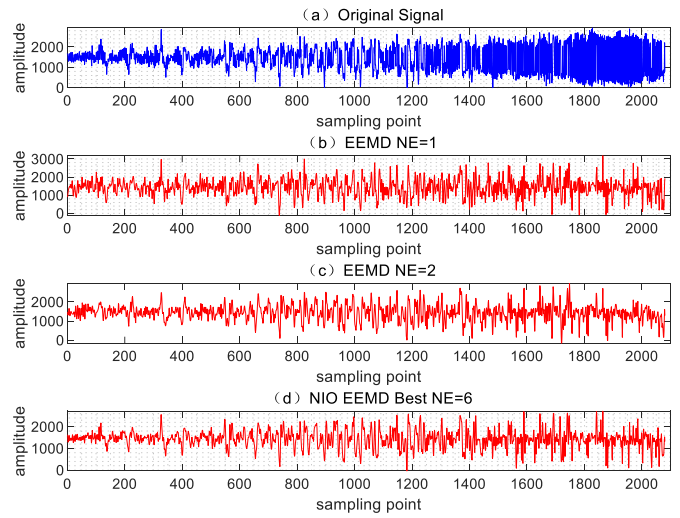

Figure 10. Comparison of Noise Reduction Effects with Different Cycle Times

In order to make a more comprehensive analysis of the above results, the performance indexes values from the first 7 cycle times of the designed algorithm were listed in Table 1.

Table 1. Performance indexes of the algorithm in different cycles 


\begin{tabular}{ccccc}
\hline SNR & \multirow{2}{*}{ SE } & R & r & $\begin{array}{c}\text { N } \\
\text { E }\end{array}$ \\
\hline 21.47 & 1491.82 & 0.5818 & 0.5320 & 1 \\
22.46 & 1490.02 & 0.6208 & 0.5913 & 2 \\
22.90 & 1495.65 & 0.6412 & 0.5956 & 3 \\
23.03 & 1492.10 & 0.6474 & 0.6053 & 4 \\
23.26 & 1493.10 & 0.6582 & 0.6156 & 5 \\
$\mathbf{2 3 . 4 1}$ & $\mathbf{1 4 9 3 . 1 4}$ & $\mathbf{0 . 6 6 4 9}$ & $\mathbf{0 . 6 2 0 9}$ & $\mathbf{6}$ \\
23.32 & 1491.17 & 0.6647 & 0.6182 & 7 \\
\hline
\end{tabular}

It can be seen from Table 1 that when the cycle times of the designed algorithm is 6 , the normalized optimization index $r$ reached its maximum value. Meanwhile, the other three indexes SNR, SE and $\mathrm{R}$ were all reached their maximum values simultaneously. When the cycle times comes to 7, the EEMD noise reduction indexes were all decreased, which means that the best noise reduction performance was achieved when $\mathrm{NE}$ equals to 6 . This is consistent with the previous results.

In order to prove that the proposed algorithm has better performance, the denoising performance of the proposed algorithm was compared with wavelet transform method and ordinary EMD method, respectively. The denoising performance form the three different methods are shown in Fig. 11.

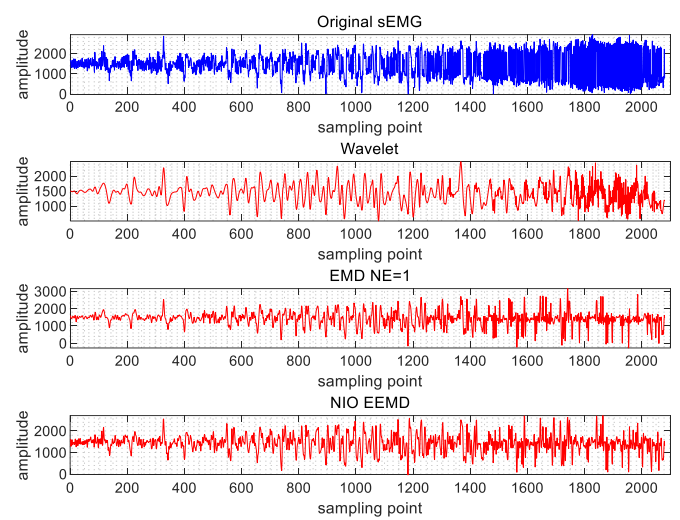

Figure 11. Denosing Performance between NIO-EEMD Method and the Other Two Methods

To prove the reliability of the experimental results statistically, the relevant noise reduction performance from the three different algorithms were listed in Table 2.

Table 2. The denosing indexes from the three algorithms

\begin{tabular}{ccccc}
\hline method & SNR & SE & R & r \\
\hline Wavelet & 21.97 & 1481.2 & 0.48 & 0.49 \\
LPF & 22.02 & 1477.3 & 0.51 & 0.50 \\
NIO & $\mathbf{2 3 . 4 1}$ & $\mathbf{1 4 9 3 . 1}$ & $\mathbf{0 . 6 6}$ & $\mathbf{0 . 6 2}$ \\
\hline
\end{tabular}

It can be seen that the best noise reduction performance achieved when the optimal times NE equals to 6 with the AIO-EEMD algorithm. With the proposed algorithm, the value of SNR index is improved from 21.47 to 23.41 . However, when adopting the $\mathrm{db} 5$ wavelet transform and EMD low-pass filtering algorithm for the same sEMG signal, the SNR index are 21.97 and 22.02, respectively. Similarly, the inverse of the $\mathrm{SE}$ and the R from the AIO-EEMD algorithm are both better than the other two algorithms for sEMG denosing. The obtained normalization search index $\mathrm{r}$ from the proposed algorithm is also the biggest among the three methods, which also proved the superiority of the proposed algorithm.

\section{CONCLUSION}

To be able to adaptively determine the numbers of components of the reconstructed signal during the denosing process, a new adaptive algorithm named AIO-EEMD is proposed in this paper. A new indicator index $r$ that combines three different denoising index parameters is defined in the algorithm. To verify the effective of the proposed algorithm, a low-cost multi-channel sEMG acquisition circuit was first designed to acquire the sEMG signal. Then, the comparative experiment were tested by using the proposed method together with the EMD low-pass filtering and wavelet transform noise reduction methods. The experimental results shown that the proposed algorithm can complete the noise reduction task without relying on experience. And the best denoising performance were got when $r$ reached its maxmium value. Also, the reduction effect obtained by this method is superior to the other two traditional methods. Thus, it can be concluded that the algorithm has good reliability.

\section{ACKNOWLEDGMENT}

The research work of this paper has been supported by 2020 Hefei University Talent Research Fund (20RC12), 2018-2019 Hefei University Talent Research Fund (18-19RC52), Major Projects of Anhui Province "R\&D and industrialization of unmanned intelligent acquisition and assembly machine" (201903a05020033), University Synergy Innovation Program of Anhui Province (GXXT-2019-048).

\section{DECLARATION OF INTEREST STATEMENT}

No conflict of interest exits in the submission of this manuscript, and it's approved by all authors for publication.

\section{References}

[1] AbuShawish, I. Y., Mahmoud S. A. "A programmable gain and bandwidth amplifier based on tunable UGBW rail-to-rail CMOS op-amps suitable for different bio-medical signal detection systems," Aeu-international journal of Electronics and Communications, vol. 141, Nov. 2021.

[2] Yang G. B., Sun Z. Y., Wang Z., et al. "Detection and identification the human surface electromyogra signal," in 2019 5th International Conference on Environmental Science and Material Application, XiAn, 2020,

[3] Hameed, H. K.; Hasan, W. Z., Shafie, S., et al. "An amplitude independent muscle activity detection algorithm based on adaptive zero crossing technique and 
mean instantaneous frequency of the sEMG signal," in Proceedings of the 2017 IEEE Regional Symposium on Micro and Nanoelectronics, Penang, pp. 183-186, Aug. 2017

[4] Xu W., Liu R., Hong X., Xiong X. "Discrimination of freshwater fish varieties based on near-infrared spectra," Transactions of the Chinese Society of Agricultural Engineering, Vol. 20, pp. 253-261, 2014.

[5] Xiong C. B., Yu L. L., Chang X. Y. "Modal parameter identification of bridge structures based on EEMD-Wavelet threshold denoising," Journal of Tianjin University, Vol. 53, pp. 378-385, Apr. 2020.

[6] Liu C. F., Zhu L. D., Ni C. B. "The chatter identification in end milling based on combining EMD and WPD," International Journal of Advanced Manufacturing Technology, Vol. 91, pp. 3339-3348, Aug. 2017.

[7] Zhang Y., Lian J., Liu F. "An improved filtering method based on EEMD and wavelet-threshold for modal parameter identification of hydraulic structure," Mechanical Systems and Signal Processing, Vol. 68, pp. 316-329, Feb. 2016.

[8] Komaty A., Boudraa A. O., Augier B., et al. "EMD-based filtering using similarity measure between probability density functions of IMFs, " IEEE Transactions on Instrumentation and Measurement, Vol. 63, pp. 27-34, Fan. 2014..

[9] Lei S., Wang K. P., Liu Z. "Development and testing of a wearable sEMG acquisition system, " IEEE Intl Conf on Dependable, Autonomic and Secure Computing, Intl Conf on Pervasive Intelligence and Computing, Intl Conf on Cloud and Big Data Computing, Intl Conf on Cyber Science and Technology Congress, Fukuoka, 2019, 527-532.

[10]Zhou Y., Fang Y. F., Gui K., et al. "sEMG Bias-driven functional electrical stimulation system for upper-limb stroke rehabilitation," IEEE Sensors Journal, Vol. 18, pp. 6812-6821, Aug. 2018.

[11]Zhou Y. X., Bi Z. Y., Ji M. J., et al. "A data-driven volitional EMG extraction algorithm during functional electrical stimulation with time variant parameters," IEEE transactions on Neural Systems and Rehabilitation Engineering, Vol. 28, pp. 1069-1080, May. 2020.

[12]Fang Y., Hettiarachchi N., Zhou D., and Liu. H. "Multi-modal sensing techniques for interfacing hand prostheses: a review, " IEEE Sensors Journal, Vol. 15, pp. 6065-6076, Nov. 2015.

[13] Xu F., Peter W. T. "A method combining refined composite multiscale fuzzy entropy with PSO-SVM for roller bearing fault diagnosis, " Journal of Central South University, Vol. 26, pp. 2404-2417, Sep. 2019.

[14] Sharma N., Prakash A., Sahi A. K., et al. "An algorithm for the estimation of the Signal-To-Noise Ratio in surface myoelectric signals generated during cyclic movement, " Instrumentation Science \& Technology, Vol. 59, pp. 219-225, Jan. 2012.

[15] Zhang K. "Research on adaptive EEMD denoising of CCD output signal for laser wavelength measurement, "
Electro-Optic Technology Application, Vol. 33, pp. 44-48, 2018.

[16] Wang H. R., Liu Z. G., Song Y., Lu X. B. "Ensemble EMD-based signal denoising using modified interval thresholding, " IET Signal Processing, Vol. 11, pp. 452-461, Jun. 2017.

[17]Johar K., Zakaria N. A. C., Ayub M. A., et al. "Comparison of EEG Data Classification between Conventional Visual Cue -Marker and EMG-Based Marker on Brain Activity," 4th international conference on system-integrated intelligence: intelligent, flexible and connected systems in products and production, Leibniz Univ Hannover, pp. 66-73, Jun. 2018.

[18] Sanchez L. E., Arias M. M., Guzman R. E., et al. "A Low-Cost EMG-Controlled Anthropomorphic Robotic Hand for Power and Precision Grasp, " Biocybernetics and Biomedical Engineering, Vol. 40, pp. 221-237, Jun. 2020.

[19]Zhao Y. Y., Zhao H., Huo X., et al. "A hybrid EMD/LPF-based denoising method for gyrowheel," Journal of Harbin Institute of Technology, Vol. 52, pp. 1-6, Jun. 2020.

[20]Cesqui B., Tropea P., Micera S., and Krebs H. I. "Emg-based pattern recognition approach in post stroke robot-aided rehabilitation: a feasibility study, " Journal of Neuroengineering and Rehabilitation, Vol. 10, pp. 127-136, Jul. 2013.

[21] Mansour, A.M.; Obeidat, M.A.; Al-Aqtash, M. "Data Mining Based Approach for Evaluation of EEG Signals for Epilepsy Detection," WSEAS Transactions on Biology and Biomedicine, Vol. 17, pp. 48-57, Jul. 2020.

[22]Zhang K, Zhang Y. J., You K., et al. "Study on the noise reduction of vehicle exhaust NOX spectra based on adaptive EEMD algorithm, "Journal of Spectroscopy, Vol. 1, pp. 1-7, Jul. 2017.

[23]Zhao Y. Y., Zhao H., Huo X. "A new multi-position calibration algorithm," Proceedings of the AIAA Guidance optimization, and Control Conference. Grapevine: AIAA, 2017.

[24] Wang Y. S., Metcalfe B., Zhao Y. Z., Zhang D. G. An assistive system for upper limb motion combining functional electrical stimulation and robotic exoskeleton[J]. IEEE Transactions on Medical Robotics and Bionics, Vol. 2, pp. 260-268, May. 2020.

\section{Authors:}

1. Wei Li, male, 1989-, Ph.D. His current research interests include control technology, big data \& signal processing.

Email: liwei@hfuu.edu.cn.

2. Wei Hu, male, 1994-, bachelor. His current research interests include switching power supply design and signal analysis.

Email: 1184296945@qq.com.

3. Kun Hu, 1998-, Master's degree candidate. His current research interests are focused on the control of permanent magnet synchronous motors.

Email: xiapenna@163.com 
4. Qiang Qin, corresponding author, male, 1966-, professor. His current research interests include application of robot technology, signal processing

Email: 1822662866@qq.com

\section{Contribution of Individual Authors to the Creation of a Scientific Article (Ghostwriting Policy)}

Wei $\mathrm{Li}$ was responsible for the framework and algorithm design of the paper.

Wei Hu carried out the design simulation and the optimization. Kun $\mathrm{Hu}$ was responsible for the data analysis and Statistics.

Qiang Qin was responsible for the English writing.

Sources of Funding for Research Presented in a Scientific Article or Scientific Article Itself

Financial supported by Hefei University Talent Research Fund (20RC12) \& (18-19RC52), 201903a05020033, GXXT-2019-048.

\section{Creative Commons Attribution License 4.0}

\section{(Attribution 4.0 International, CC BY 4.0)}

This article is published under the terms of the Creative Commons Attribution License 4.0

https://creativecommons.org/licenses/by/4.0/deed.en US 